\title{
Irinotecan-Induced Transient Dysarthria: Case Series and Updated Literature Review
}

\author{
Mariagrazia De Lisa - Zelmira Ballatore - Giulia Marcantognini · \\ Chiara Pierantoni - Stefania Antognoli · Mirco Pistelli • \\ Alessandra Pagliacci · Rossana Berardi
}

Received: October 23, 2019 / Published online: January 6, 2020

(C) The Author(s) 2020

\begin{abstract}
Irinotecan-based regimens are used worldwide for the treatment of several recurrent or advanced gastrointestinal malignancies. In this paper we describe the cases of four patients treated in our institution who developed acute dysarthria while receiving intravenous infusion of irinotecan. In all our cases, dysarthria occurred during the infusion of the first course of irinotecan, and then resolved rapidly without any sequelae. Imaging of the brain was performed, but failed to show any evidence of an acute neurological event. We also reviewed the literature on this very uncommon adverse event. The pathogenesis of irinotecan-induced dysarthria is still unknown and is not completely elucidated by the current pharmacodynamic or kinetic explanations; therefore, we could only hypothesize some assumptions.
\end{abstract}

Enhanced Digital Features To view enhanced digital features for this article go to https://doi.org/10.6084/ m9.figshare.11371914.

M. De Lisa · Z. Ballatore · G. Marcantognini ·

C. Pierantoni · S. Antognoli · M. Pistelli .

A. Pagliacci $\cdot$ R. Berardi $(\bowtie)$

Medical Oncology, Azienda Ospedaliero-

Universitaria Ospedali Riuniti-Ancona, Università

Politecnica delle Marche, Ancona, Italy

e-mail: r.berardi@univpm.it
Keywords: Adverse events; Dysarthria; Neurological side effects; Rinotecan

\section{Key Summary Points}

Irinotecan-based chemotherapy regimens are used worldwide in the treatment of recurrent or advanced gastrointestinal (GI) cancers, in combination with other cytotoxic drugs or monoclonal antibodies. The most common side effects include acute cholinergic syndrome (ACS) mediated through the inhibition of acetylcholinesterase (AchE), delayed diarrhoea, nausea and vomiting, myelosuppression, alopecia, stomatitis and abdominal pain.

Dysarthria, defined as an impairment of speech caused by an alteration of the strength and control of speech muscles, is a very uncommon central nervous system (CNS) adverse event occurring rarely during or shortly following irinotecan (CPT-11) infusion. Its pathogenesis is not completely elucidated by current pharmacodynamic or kinetic explanations. In all the cases previously reported in the literature, it occurred during the first cycle within 90 min of drug infusion, and was not accompanied by any other signs on clinical examination and imaging. 
Dysarthria was transient and resolved within $24 \mathrm{~h}$ independently of any adopted strategies.

The aim of our study is to add to the scientific literature our institution's experience of four patients who experienced transient dysarthria related to CPT-11. We also performed a literature review reporting the different pathogenic mechanisms that have been previously hypothesized by several authors, in terms of pharmacodynamic or pharmacokinetic explanations.

In order to better understand the pathogenesis of this rare adverse event, we propose to investigate the potential role of UGT1A1 gene polymorphisms, which are also related to an increased rate of severe haematological and/or gastrointestinal toxicities.

Lastly, we remind the importance of being aware of the recommended guidelines about the irinotecan administration (in terms of preparation of infusion solution and pre-medication with antiemetic drugs, steroids and atropine) and we highlighted the need to agree recommendations and/or protocols regarding the management of neurological adverse events.

\section{INTRODUCTION}

Irinotecan (CPT-11) is a topoisomerase I inhibitor that has shown anti-tumor activity in several solid tumors. CPT-11-based chemotherapy regimens approved up to now are used in the treatment of recurrent or advanced colorectal, gastric, and pancreatic cancer [1-5]. Furthermore, in metastatic colon-rectal cancer, CPT-11 has shown activity in both first- and second-line settings, even in combination with biological agents such as cetuximab [6, 7], panitumumab [8], and bevacizumab [9].

The most common side effects include acute cholinergic syndrome (ACS), delayed diarrhoea, nausea and vomiting, and myelosuppression. Other minor toxicities include alopecia, stomatitis, and abdominal pain [10]. It has been suggested that the cholinergic side effects of irinotecan are mediated through the inhibition of acetylcholinesterase (AchE). Rapid reversibility of the inhibition of AchE by CPT-11 and lower activity of the carboxylate form may explain the transient nature of the clinically observed cholinergic toxicity [11]. Symptoms may occur shortly after the beginning of infusion and/or hours after administration; they are generally short-lived and rarely life-threatening. Co-administration of atropine $0.2 \mathrm{mg}$ administered subcutaneously may prevent these adverse reactions.

Some central nervous system (CNS) adverse events related to CPT-11 have also been described, including dysarthria and/or aphasia, and to date only 29 cases have been reported in the literature [12-21, 27] (Table 1). The mechanism of CPT-11-related neurological toxicity is not completely understood. The aim of this paper is to describe four consecutive clinical cases of patients treated in our institution who developed dysarthria as an acute neurological sign secondary to CPT-11 administration, and to review the literature data.

\section{CASE PRESENTATIONS}

\section{Patient 1}

A 49-year-old man diagnosed with locally advanced rectal cancer at the end of 2006 was treated with neoadjuvant radiotherapy combined with capecitabine followed by surgery in February 2007.

From April to July 2007 he received adjuvant chemotherapy (12 cycles according to FOLFOX6 schedule); grade 1-2 haematological toxicity, according to the Common Terminology Criteria for Adverse Events (CTCAE) v 4.0, was reported. In June 2009, thoracic abdominal and pelvic computed tomography (CT) scan showed a right lung metastasis and a suspected local relapse.

Molecular analysis of K-RAS showed no mutation (wild-type); therefore the patient 


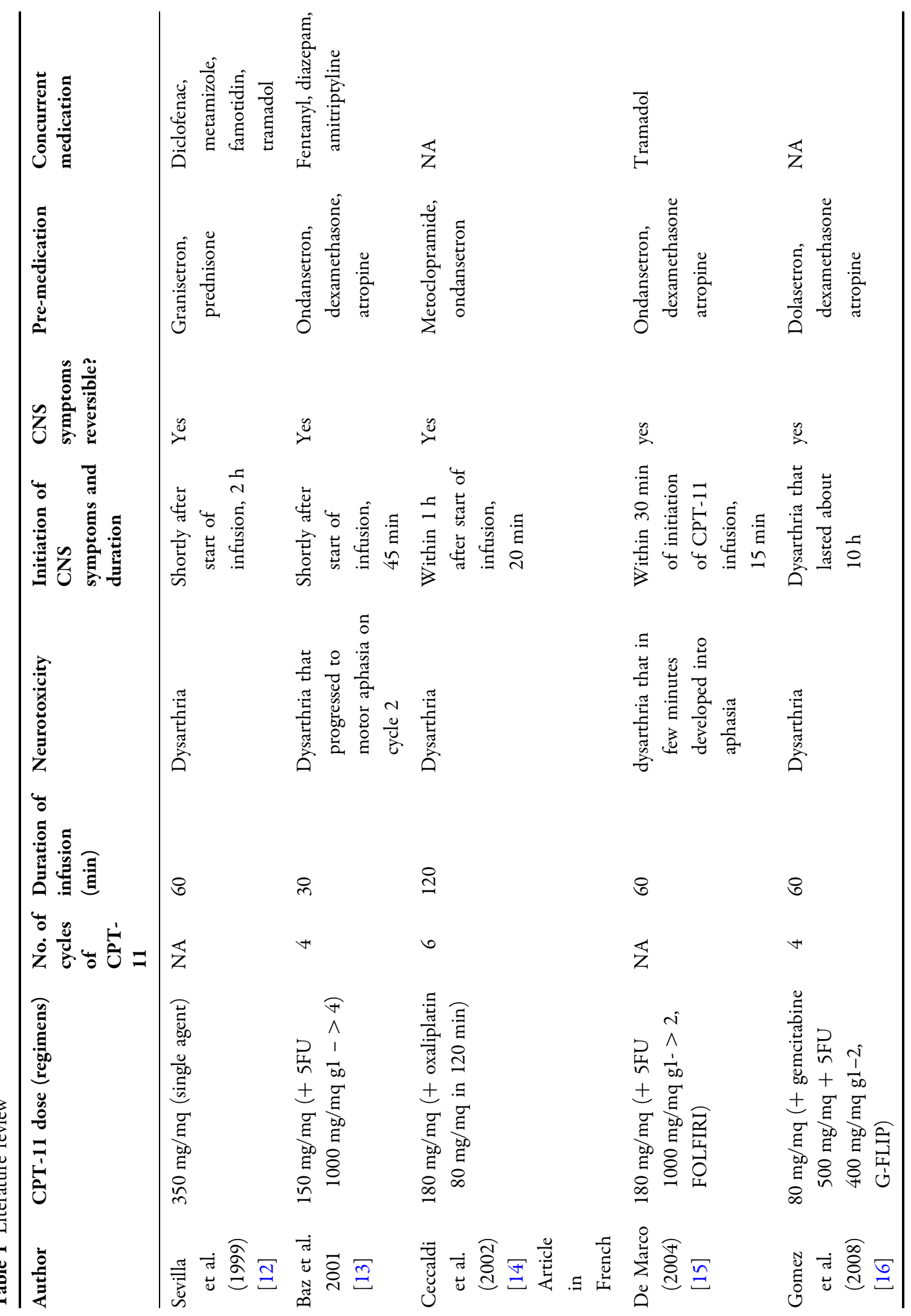




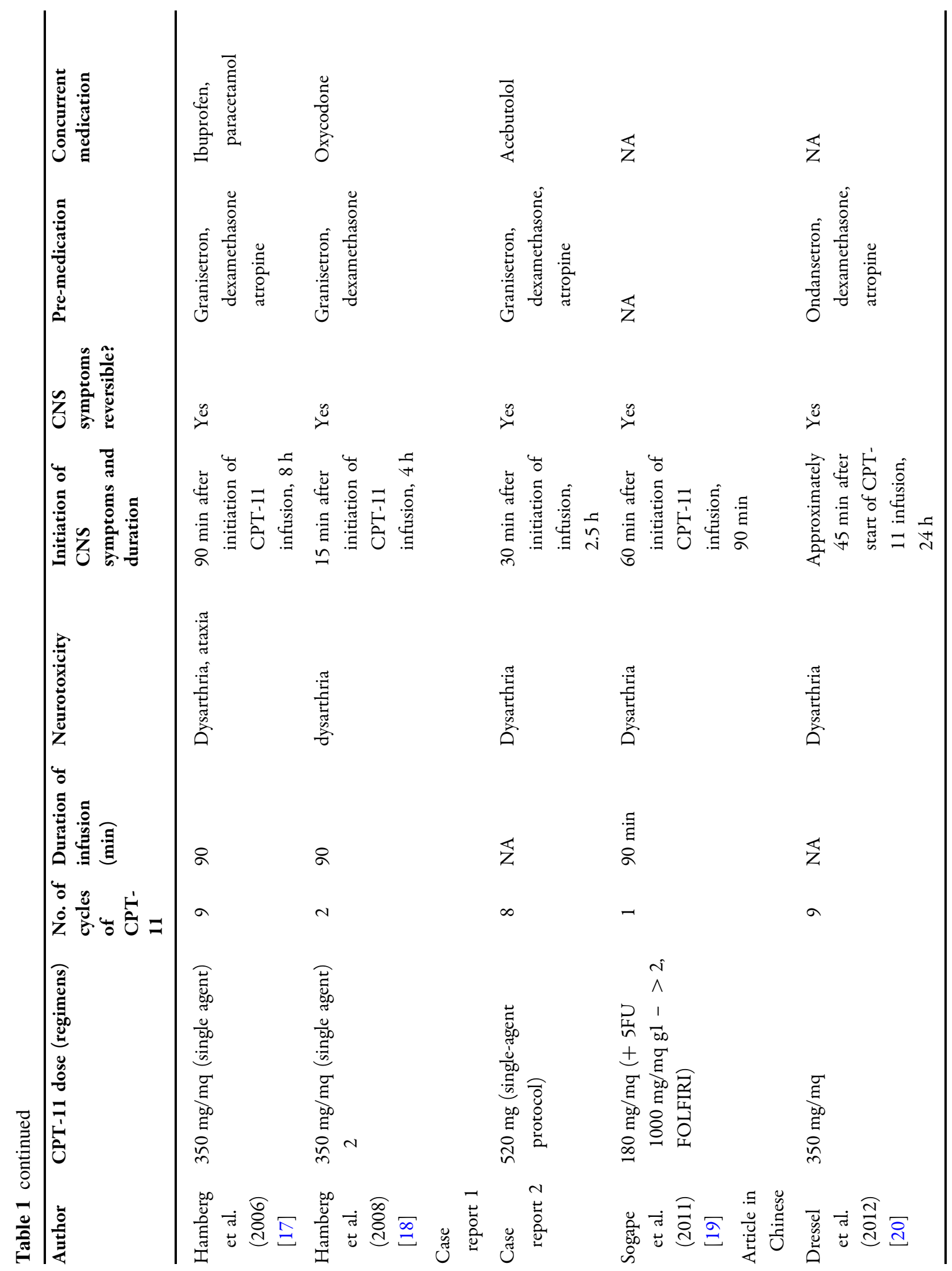




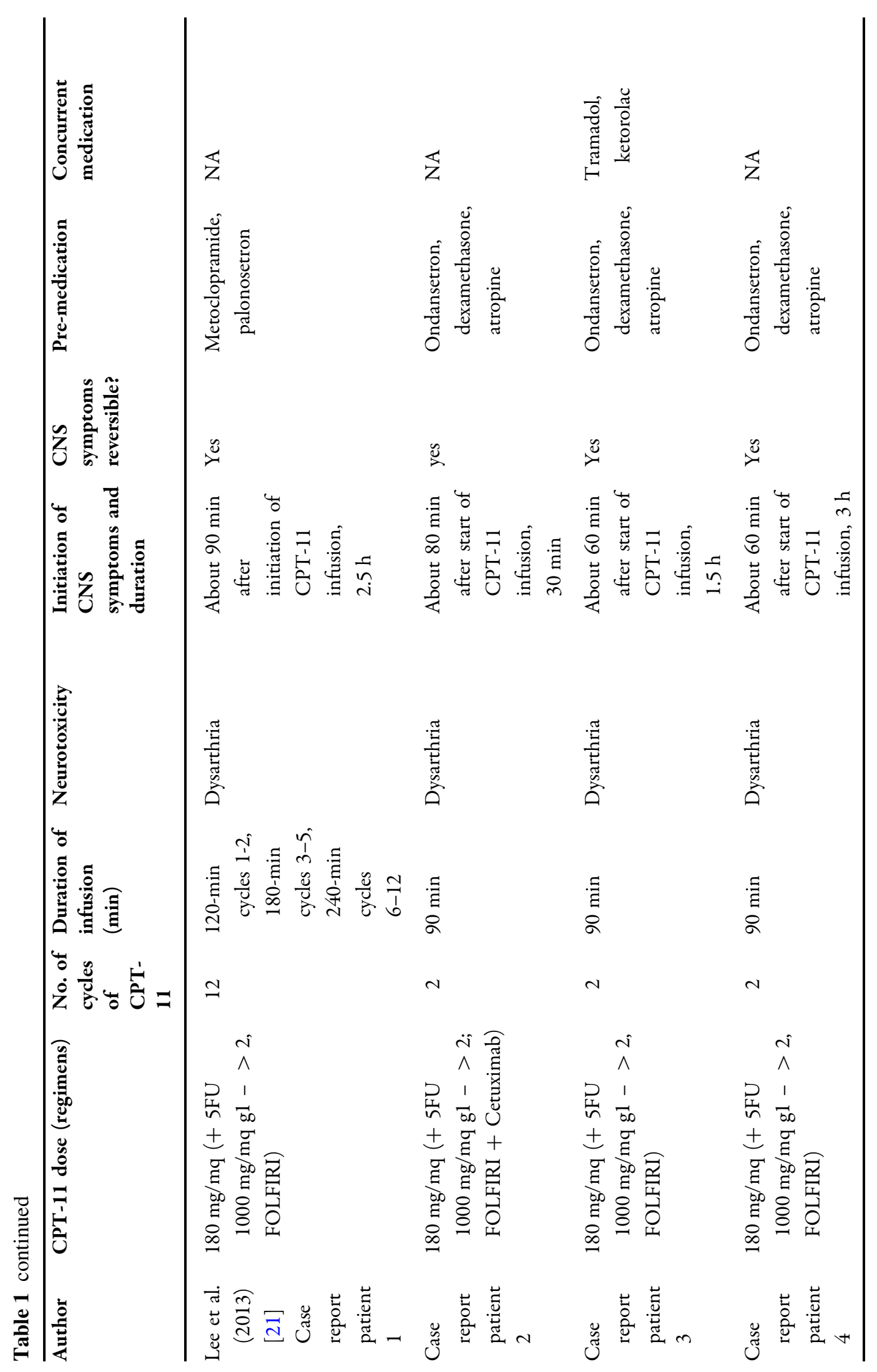




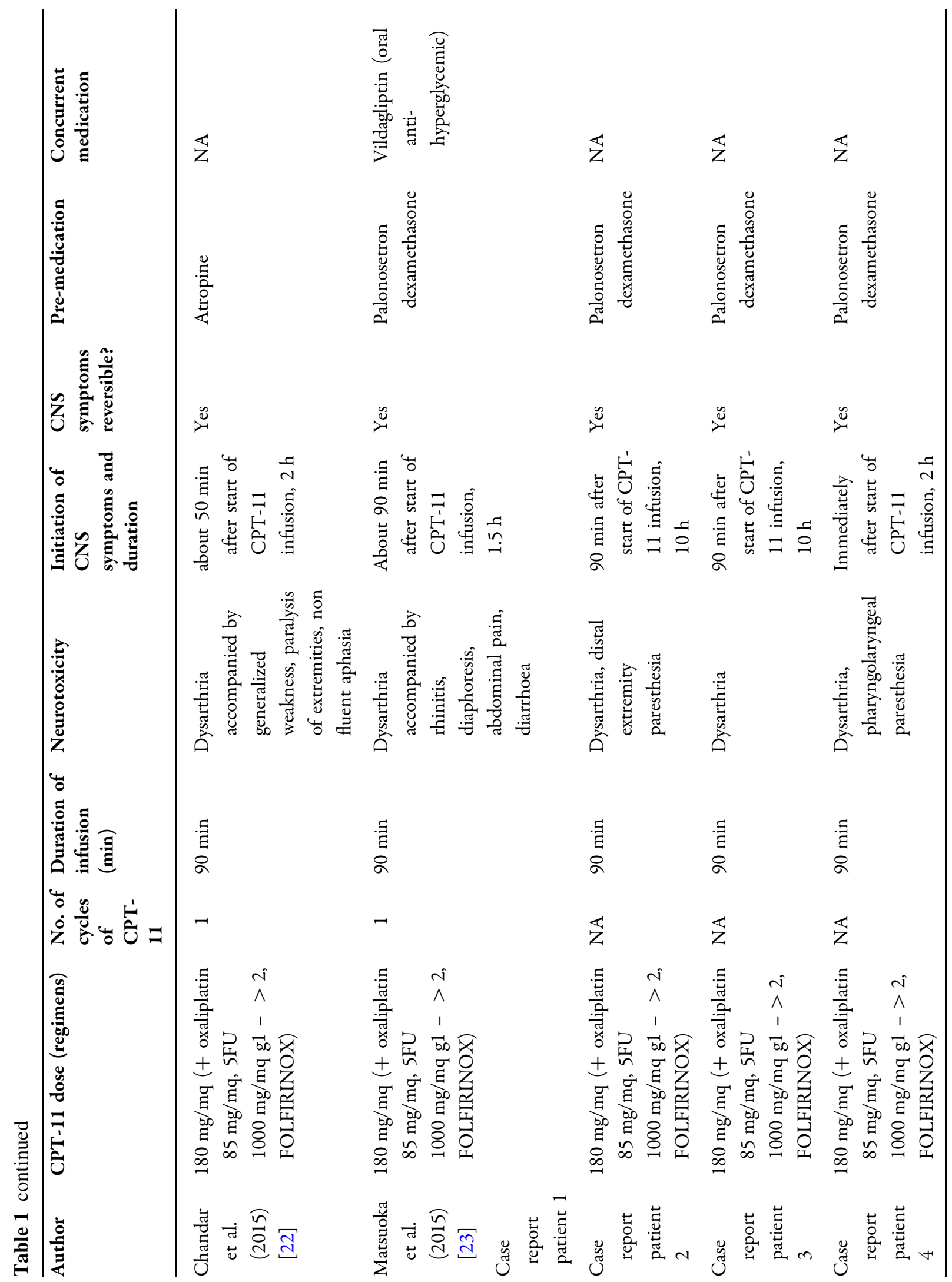




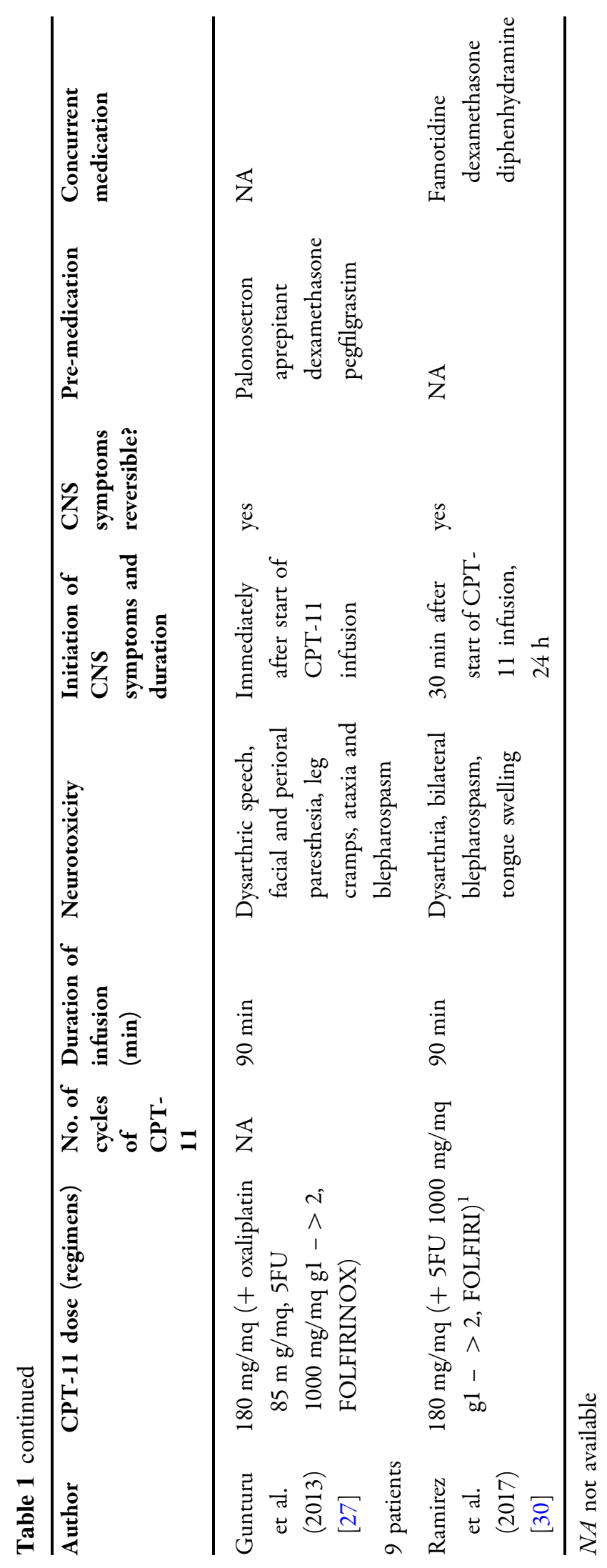


started first-line chemotherapy with irinotecan $180 \mathrm{mg} / \mathrm{m}^{2}$, folinic acid $200 \mathrm{mg} / \mathrm{m}^{2}$, and fluorouracil (5FU) bolus $400 \mathrm{mg} / \mathrm{m}^{2}$ followed by infusion of fluorouracil $2400 \mathrm{mg} / \mathrm{m}^{2}$ over $46 \mathrm{~h}$ ) (FOLFIRI regimen) every 14 days, combined with cetuximab $\left(400 \mathrm{mg} / \mathrm{m}^{2}\right.$ for the first infusion and $250 \mathrm{mg} / \mathrm{m}^{2}$ thereafter). No relevant comorbidities had been reported; the only concomitant medication was sertraline for anxiety. The patient's complete blood count and biochemical test results before the start of chemotherapy were within normal ranges. According to the protocol, CPT-11 was administered as intravenous infusion over $90 \mathrm{~min}$ after subcutaneous atropine $(0.25 \mathrm{mg})$ and intravenous antiemetics (ondansetron and dexamethasone).

About 80 min after the start of CPT-11 infusion, the patient developed dysarthria, which completely resolved within $30 \mathrm{~min}$ without administering any medication. The patient was conscious and alert, and physical and neurological examinations showed no abnormalities. Dysarthria rapidly regressed without any sequelae.

During the second cycle, before starting irinotecan infusion, the patient received double doses of subcutaneous atropine $(0.5 \mathrm{mg})$, with the aim of preventing neurological toxicity. Similarly to the first cycle, the patient developed dysarthria at the end of CPT-11 infusion, and the symptom definitely resolved after $30 \mathrm{~min}$.

Although neurological toxicity seems to be reversible and not dose-limiting, considering the uncertainty in data regarding CNS side effects related to irinotecan, we decided to discontinue irinotecan.

That patient started a new chemotherapy regimen, with partial response of lung metastases; no neurological reaction during or after the infusion of chemotherapy was reported. In December 2009 he underwent positron emission tomography (PET-CT) imaging, which confirmed localized lung disease, and he therefore underwent lung resection. After surgery, the patient asked to be referred to another institution closer to his home and was lost to follow-up.

\section{Patient 2}

A 58-year-old man was diagnosed with locally advanced pancreatic adenocarcinoma in October 2013. The patient had a good performance status score according to the Eastern Cooperative Oncology Group (ECOG) scale, and started neoadjuvant FOLFIRINOX chemotherapy (oxaliplatin $85 \mathrm{mg} / \mathrm{m}^{2}$, irinotecan $180 \mathrm{mg} / \mathrm{m}^{2}$, leucovorin $400 \mathrm{mg} / \mathrm{m}^{2} \mathrm{IV}$, and $5 \mathrm{FU} 2400 \mathrm{mg} / \mathrm{m}^{2} \mathrm{IV}$ by continuous infusion over $48 \mathrm{~h}$, with dexamethasone $10 \mathrm{mg}$ and ondansetron $12 \mathrm{mg}$ IV as pre-medication). According to the schedule, irinotecan was administered as an intravenous infusion over $90 \mathrm{~min}$ immediately after oxaliplatin. Subcutaneous atropine $(0.25 \mathrm{mg})$ was given for cholinergic syndrome prophylaxis.

About an hour after the start of the irinotecan infusion, the patient developed slurred speech that quickly progressed to dysarthria. CPT-11 infusion was quickly interrupted and symptoms spontaneously decreased and then completely resolved in about $90 \mathrm{~min}$. At clinical examination, he was conscious and alert, neither motor nor sensitive neurological signs were noted, and the patient did not report any other symptoms. Two hours later, we decided to restart the infusion of irinotecan, slowing the infusion rate, without any adverse event. The second cycle was administered maintaining the same dose and with the same pre-medication, and the patient did not show dysarthria or other neurological symptoms. After the second cycle, he developed grade 2 thrombocytopenia according to the CTCAE ( $\mathrm{v}$ 4.03) and he interrupted CPT-11, continuing the FOLFOX regimen. A partial response in the liver was shown; therefore, he underwent a partial hepatectomy. The patient is alive and disease-free; we closely monitor him with routine CT scans.

\section{Patient 3}

In May 2012, a 60-year-old man underwent right hemicolectomy for adenocarcinoma of the right colon. The pathology report showed poorly differentiated adenocarcinoma of the ileocecal valve (stage pT3 N2 M0) carrying a 
K-RAS mutation at position 12 [KRAS c.35G > A (G12D)].

From June to August 2012, the patient received adjuvant chemotherapy according to the FOLFOX-6 schedule. After four cycles, he experienced rapidly progressive clinical deterioration and a significant increase in Ca 19.9.

Due to the severe and fast progression of the disease, the patient started a second-line treatment with CPT-11 $180 \mathrm{mg} / \mathrm{m}^{2}$, folinic acid $200 \mathrm{mg} / \mathrm{m}^{2}$, and $5 \mathrm{FU}$ bolus $400 \mathrm{mg} / \mathrm{m}^{2}$ followed by infusion of $5 \mathrm{FU} 2400 \mathrm{mg} / \mathrm{m}^{2}$ over $46 \mathrm{~h}$ every 14 days according to the FOLFIRI regimen. He was consuming low doses of opioids for diseaserelated abdominal pain. The patient received the first cycle of FOLFIRI without experiencing any related adverse event. During the second infusion of CPT-11, about $60 \mathrm{~min}$ after the start, he developed dysarthria which evolved to aphasia. Irinotecan infusion was promptly interrupted and the patient was hospitalized for the following $48 \mathrm{~h}$. Neurological symptoms spontaneously resolved within $7 \mathrm{~h}$ after the admission. The CNS CT scan performed in emergency did not show any metastasis or signs of acute ischemic/hemorrhagic event. In the following weeks, the patient's clinical condition deteriorated rapidly, with evidence of hemorrhagic ascites related to progressive disease. He then started best supportive care and died about 1 month later.

\section{Patient 4}

A 36-year-old woman underwent right hemicolectomy due to adenocarcinoma in July 2009. The pathological stage at diagnosis was pT3N1M0. After surgery, the patient received adjuvant chemotherapy according to the FOLFOX-6 regimen for a total of 12 cycles. Nearly $4 \mathrm{~h}$ after the end of the last oxaliplatin infusion, she experienced pharyngolaryngeal spasm associated with moderate dyspnoea, which resolved after IV steroid infusion. A CT scan performed at the end of adjuvant chemotherapy showed disease recurrence; in fact a single small lump in the inferior lobe of the right lung was documented. Referred to the thoracic surgeon, in March 2010 the patient underwent radical wedge resection; the pathology report confirmed that the lesion was related to the primary colic carcinoma. The patient was followed up for 6 years with no evidence of recurrent disease, until a month ago when she developed a persistent cough. We performed a thorax-abdomen CT scan with contrast, which showed metastatic disease to the mediastinum, lungs, adrenal glands, and liver. The case of this young lady was discussed extensively within our multidisciplinary tumor board: since the disease relapse shown in 2010 had occurred during the adjuvant oxaliplatin-based chemotherapy and, most importantly, at the end of that, she probably had developed an adverse event which could be interpreted as hypersensitive reaction or neuropathic toxicity. Therefore, we decided to start first-line chemotherapy with $5 \mathrm{FU}$ and irinotecan according to the FOLFIRI regimen, and molecular analysis of pan-RAS was performed. During the first cycle, about $45 \mathrm{~min}$ after the start of CPT-11, the patient developed dysarthria. Irinotecan infusion was promptly stopped, and a bolus of $1 \mathrm{~g}$ hydrocortisone sodium succinate IV and $1 \mathrm{mg}$ of subcutaneous atropine were given. Dysarthria regressed completely in about half an hour. Similarly to the aforementioned cases, no other signs were noted at clinical examination. The patient was hospitalized for administration of the second cycle, and the infusion rate of irinotecan was doubled to $180 \mathrm{~min}$. A double dose of atropine $(1 \mathrm{~g})$ and dexamethasone $(16 \mathrm{mg})$ were given as prophylaxis. Although the young patient was very anxious, aware of the limited number of available therapeutic strategies, she did not experience any acute adverse event during or after the irinotecan infusion. We decided to combine an anti-EGFR antibody with chemotherapy at the subsequent cycle, as her adenocarcinoma was found to be wild-type for N-RAS, K-RAS, and B-RAF.

\section{Compliance with Ethics Guidelines}

This study conformed with the Helsinki Declaration of 1964 , as revised in 2013, concerning human and animal rights. All patients gave their written consent to all the diagnostic- 
therapeutic procedures. Ethical committee approval was not required, as per country law.

\section{DISCUSSION}

Dysarthria is defined as an impairment of speech caused by an alteration of the strength and control of speech muscles. It is often characterized by slurred or slow speech that makes it difficult to be understood. Common causes of dysarthria include neurological disorders such as stroke, brain injury, brain tumors, and some conditions that cause facial paralysis or tongue or throat muscle weakness. Dysarthria may also be induced by medications, including cytotoxic drugs, such as CPT-11, methotrexate, fluoropyrimidine, and oxaliplatin [22].

To date, 29 cases of transient dysarthria and/ or aphasia observed during or shortly following the administration of irinotecan have been reported in the literature and are summarized in Table $1[12-21,27]$.

Transient dysarthria is not reported as a side effect in the prescribing information sheet, although it is described as an unpredictable adverse reaction of irinotecan seen in the postmarketing phase (see Table 2).

CNS toxicity most commonly occurred during the first cycle of irinotecan, although it may occur in subsequent cycles, within $90 \mathrm{~min}$ of drug infusion and despite the co-administration of atropine or other medications. The pathogenesis and the specific mechanism involved in the development of CNS symptoms remain unclear.

Some authors reported possible resolution with atropine administration, suggesting a relationship between CPT-11-induced transient dysarthria and anticholinesterase inhibition.

Nevertheless, although the majority of neoplastic patients did not have macroscopic CNS metastases, they generally showed an alteration of the blood-brain barrier (BBB), which might be related to an increase in levels of CPT-11 or its active metabolite SN38 in the cerebrospinal fluid. However, cytotoxic oedema and altered systemic clearance of irinotecan did not seem to be involved [19].
In all reported cases [12-21], clinical signs were stable and neurological examinations showed no evidence of neurological abnormalities, except dysarthria. Patients were monitored carefully and observed to return close to their baseline status within 1-2 h.

In most of the cases, imaging of the brain, such as magnetic resonance imaging (MRI) or CT scan, were also performed, but they failed to show any evidence of stroke or other acute CNS abnormalities, even in diffusion-weighted images. In Dressel's case report [18], after neurological assessment and MRI imaging, an acoustic analysis was performed by an experienced speech therapist and otorhinolaryngologist that showed reduced ability in fine-tuning of motor functions of the tip of the tongue and a minimal reduction in the power of speech at labiodental contact. A reduction in sound coordination was then found for consonants generated by the tip of the tongue. However, it was not possible to identify the anatomical level of these symptoms; it was likely central, at the level of brain stem.

To the best of our knowledge, the pathogenesis of irinotecan-induced dysarthria is still unknown, and thus we can only hypothesize some assumptions.

Plasma concentration can be described by a biphasic model including a first peak within $2 \mathrm{~h}$ after the start of CPT-11 infusion, followed by a declining phase: mean terminal elimination half-life of CPT-11 and SN-38 is about $14.2 \mathrm{~h}$ and $13.8 \mathrm{~h}$, respectively $[16,17,23]$.

In an earlier study, Hamberg [17] demonstrated that there were no differences in plasma concentrations of CPT-11 or its active metabolite $\mathrm{SN}-38$ between patients developing the dysarthria and the larger population, so the isolated dysarthria cannot be explained by differences in systemic clearance of irinotecan. Further, there is a lack of an apparent relationship between the infusion rate and dose of CPT11 and the onset of dysarthria and its duration.

In all reported literature data, patients developed dysarthria early during the infusion, thus suggesting the utility of implementing intensive nursing care in order to stop the infusion promptly at the onset of symptoms. Moreover, dysarthria occurred independently of 


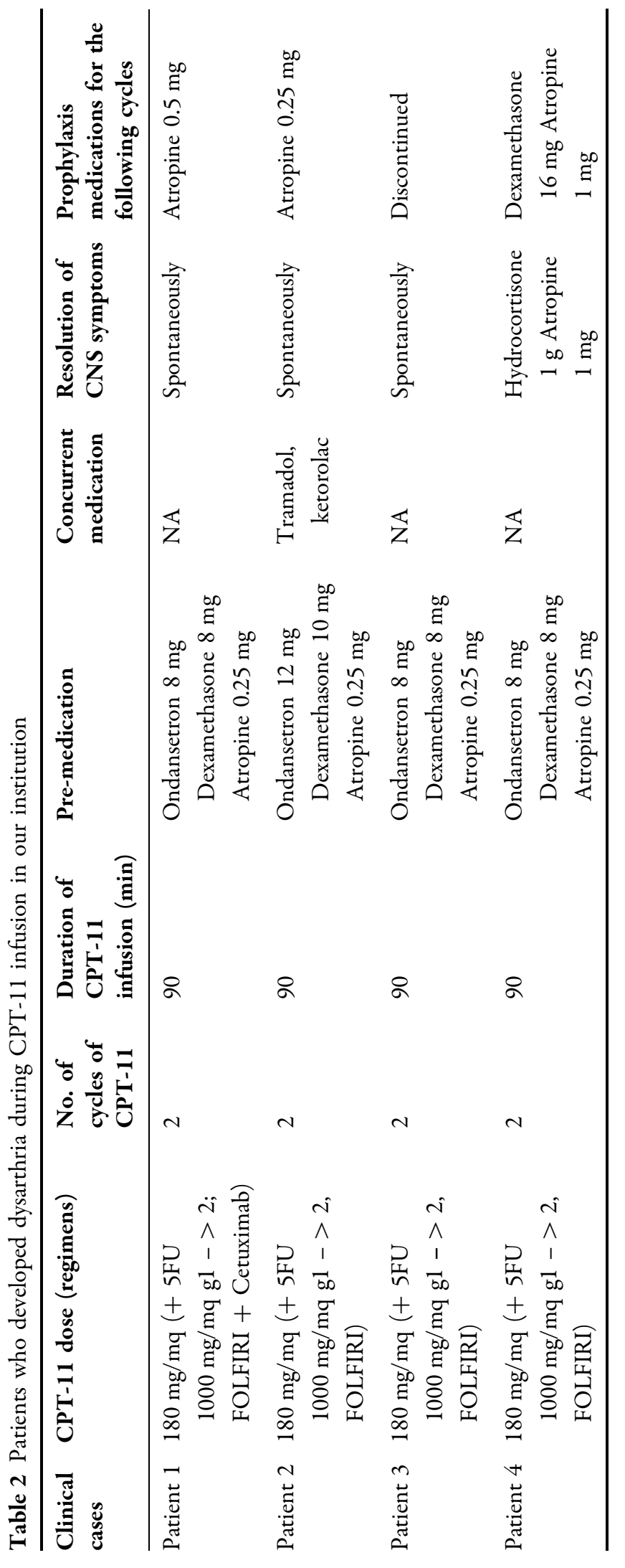


pre-medication given before treatment (antiemetic drugs, steroids, atropine) and concurrent medication (nonsteroidal anti-inflammatory drugs, opioids, others).

Dysarthria appeared as a reversible symptom within $24 \mathrm{~h}$, and quick resolution was described in all reported cases, ranging from 2 to $7 \mathrm{~h}$. Resolution was also independent of the strategies adopted, such as discontinuing CPT-11 infusion, reducing the CPT-11 infusion rate or dosage [17], or administering further atropine dose or some intravenous fluids [19].

All patients received pre-medication with atropine $1 \mathrm{mg}$ and 5-hydroxytryptamine (serotonin) receptors inhibitors, but their possible influence is unknown.

Interestingly, no cases of dysarthria were reported in the clinical trials of FOLFOXIRI in advanced colorectal cancer [24-26], in which irinotecan was administered prior to oxaliplatin. Conversely, an unexpected high incidence of dysarthria was reported in a retrospective analysis and in a phase II trial from Japan [27, 28]. Based on these data, oxaliplatin co-administration seems to increase the cholinergic effects of irinotecan, making dysarthria more evident. Furthermore, some authors speculate that FOLFIRINOX-induced dysarthria is associated with the sequence of drug administration (i.e., intravenous infusion of oxaliplatin, immediately followed by irinotecan) [21]. Moreover, it is well known that patients with reduced UDP-glucuronosyltransferase (UGT) 1A1 activity, including those who are homozygous for the UGT1A1*28 allele (UGT1A1 7/7 genotype) or those heterozygous for that allele (UGT1A1 6/7 genotype), show an increased risk of developing haematological and/or gastrointestinal toxicities compared with those patients who are homozygous for the wild-type allele (UGT1A1 6/6 genotype). Irinotecan dose reduction is thus recommended in homozygous ${ }^{*} 28 /{ }^{*} 28$ patients. Neither clinical response nor prognosis was found to be significantly associated with UGT1A1 gene polymorphisms in patients with advanced colorectal cancer [29].

Considering this very uncommon type of toxicity (acute neurological symptoms), it might be interesting to investigate the specific polymorphism in the UGT1A1 carried by those patients. Although the exact pathogenesis of the irinotecan-induced dysarthria is still unknown or not completely elucidated by the current pharmacodynamic or kinetic explanations, recommendations and/or protocols regarding the management of neurological adverse events related to irinotecan should be established.

Guidelines recommend administration of pre-medication with antiemetic agents, including dexamethasone given with 5HT3 blocker (e.g., ondansetron or granisetron), on the day of treatment, starting at least $30 \mathrm{~min}$ before irinotecan administration.

All dose modifications should be based on the worst preceding toxicity according to the CTCAE (v 4.03).

Regarding the preparation of the infusion solution, it is recommended that CPT-11 be diluted in $5 \%$ dextrose (preferred) or $0.9 \%$ sodium chloride to a final concentration range of $0.12-2.8 \mathrm{mg} / \mathrm{mL}$ prior to infusion.

Prophylactic or therapeutic administration of $0.25-1 \mathrm{mg}$ of intravenous or subcutaneous atropine is suggested (unless clinically contraindicated) to prevent diarrhoea and cholinergic reactions.

\section{CONCLUSION}

Acute neurological events (dysarthria, severe generalized weakness, paralysis, and aphasia) reported in the literature should be carefully highlighted in the prescribing information sheet as rare but potentially severe adverse events. Further diagnostic tests should be performed in those patients who develop dysarthria and other neurological toxicities during irinotecan infusion, based on the magnitude and the duration of the symptoms, to exclude any potential acute CNS events.

\section{ACKNOWLEDGEMENTS}

We thank the participants of the study. 
Funding. No funding or sponsorship was received for publication of this article.

Authorship. All named authors meet the International Committee of Medical Journal Editors (ICMJE) criteria for authorship for this article, take responsibility for the integrity of the work as a whole, and have given their approval for this version to be published.

Disclosures. Mariagrazia De Lisa, Zelmira Ballatore, Giulia Marcantognini, Chiara Pierantoni, Stefania Antognoli, Mirco Pistelli, and Alessandra Pagliacci have nothing to disclose. Rossana Berardi is the Editor-in-Chief of this journal but has no other relevant disclosures.

Compliance with Ethics Guidelines. This study conformed with the Helsinki Declaration of 1964, as revised in 2013, concerning human and animal rights. All patients gave their written consent to all the diagnostic-therapeutic procedures. Ethical committee approval was not required, as per country law.

Data Availability. All data generated or analyzed during this study are included in this published article.

Open Access. This article is distributed under the terms of the Creative Commons Attribution-NonCommercial 4.0 International License (http://creativecommons.org/licenses/ by-nc/4.0/), which permits any noncommercial use, distribution, and reproduction in any medium, provided you give appropriate credit to the original author(s) and the source, provide a link to the Creative Commons license, and indicate if changes were made.

\section{REFERENCES}

1. Douillard JY, Cunningham D, Roth $\mathrm{AD}$, et al. Irinotecan combined with fluorouracil compared with fluorouracil alone as first-line treatment for colorectal cancer: a multicenter randomized trial. Lancet. 2000;355:1041-7.
2. Saltz LB, Cox JV, Blanke C, et al. Irinotecan plus fluorouracil and leucovorin for metastatic colorectal cancer. N Engl J Med. 2000;343:905-14.

3. Yoo C, Hwang JY, Kim JE, et al. A randomised phase II study of modified FOLFIRI.3 vs modified FOLFOX as second-line therapy in patients with gemcitabine-refractory advanced pancreatic cancer. $\mathrm{Br} \mathrm{J}$ Cancer. 2009;101(10):1658-63.

4. Conroy T, Desseigne F, Ychou M, et al. FOLFIRINOX versus gemcitabine for metastatic pancreatic cancer. N Engl J Med. 2011;364:1817-25.

5. Dank M, Zaluski J, Barone C, et al. Randomized phase III study comparing irinotecan combined with 5-fluorouracil and folinic acid to cisplatin combined with 5-fluorouracil in chemotherapy naive patients with advanced adenocarcinoma of the stomach or esophagogastric junction. Ann Oncol. 2008;19(8):1450-7.

6. Sobrero AF, Maurel J, Fehrenbacher L, et al. EPIC: phase III trial of cetuximab plus irinotecan after fluoropyrimidine and oxaliplatin failure in patients with metastatic colorectal cancer. J Clin Oncol. 2008;26(14):2311-9.

7. Van Cutsem E, Köhne C-H, Hitre E, et al. Cetuximab and chemotherapy as initial treatment for metastatic colorectal cancer. $\mathrm{N}$ Engl J Med. 2009;360:1408-17.

8. Peeters M, Price TJ, Cervantes A, et al. Randomized phase III study of panitumumab with fluorouracil, leucovorin, and irinotecan (FOLFIRI) compared with FOLFIRI alone as second-line treatment in patients with metastatic colorectal cancer. J Clin Oncol. 2010;28(31):4706-13.

9. Hurwitz H, Fehrenbacher L, Novotny W, et al. Bevacizumab plus irinotecan, fluorouracil, and leucovorin for metastatic colorectal cancer. N Engl J Med. 2004;350:2335-42.

10. Wiserman LR, Markham A. Irinotecan A. review of its pharmacological properties and clinical efficacy in the management of advanced colorectal cancer. Drugs. 1996;52:606-23.

11. Dodds HM, Rivory LP. The mechanism for the inhibition of aceytilcholinesterase by irinotecan (CPT-11). Mol Pharmacol. 1999;56(6):1346-53.

12. Sevilla Garcia I, Rueda A, Alba E. Irinotecan-induced central nervous system toxicity: a case report. J Natl Cancer Inst. 1999;91(7):647.

13. Baz DV, Bofill JS, Nogueira JAM. Irinotecan-Induced dysarthria. J Nat Cancer Inst. 2001;18:1419-20. 
14. Ceccaldi B, Kara F, Mommeja-Marin H, et al. Dysarthria during irinotecan administration. Rev Med Interne. 2002;23(11):950-1.

15. De Marco S, Squilloni E, Vigna L, Bertagnolio MF, Sternberg CN. Irinotecan chemotherapy associated with transient dysarthria and aphasia. Ann Oncol. 2004;15:1147-8.

16. Gomez JA, Sanchez I, Ramirez JA. Irinotecan-induced dysarthria: an insight into its pathogenesis? Case Rep GI Oncol. 2008;2:209-10.

17. Hamberg P, De Jong FA, Brandsma D, Verweij J, Sleijfer S. Irinotecan-induced central nervous system toxicity. Report on two cases and review of the literature. Acta Oncol. 2008;47(5):974-8.

18. Dressel AJ, van der Mijn JC, Aalders IJ, Rinkel RN, van der Vliet HJ. Irinotecan-induced dysarthria. Case Rep Oncol. 2012;5(1):47-51.

19. Lee KA, Kang HW, Ahn JH, Suk HJ, Kim H. Dysarthria induced by irinotecan in a patient with colorectal cancer. Am J Health Syst Pharm. 2013;70(13):1140-3.

20. Chandar M, de Wilton Marsh R. Severe generalized weakness, paralysis, and aphasia following administration of irinotecan and oxaliplatin during FOLFIRINOX chemotherapy. Case Rep Oncol. 2015;8: 138-41.

21. Matsuoka A, Maeda O, Inada-Inoue M, et al. FOLFIRINOX-induced reversible dysarthria: a case report and review of previous cases. Oncology Letters. 2015;10:2662-4.

22. Joseph R, Dasanu CA. A curious case of oxaliplatininduced neurotoxicity: recurrent, self-limiting dysarthria. J Oncol Pharm Pract. 2014;20(5):393-6.

23. Abigerges D, Chabot GG, Armand JP, Hérait P, Gouyette A, Gandia D. Phase I and pharmacologic studies of the camptothecin analog irinotecan administered every 3 weeks in cancer patients. J Clin Oncol. 1995;13(1):210-21.

24. Falcone A, Ricci S, Brunetti I, et al. Phase III trial of infusional fluorouracil, leucovorin, oxaliplatin and irinotecan (FOLFOXIRI) compared with infusional fluorouracil, leucovorin and irinotecan (FOLFIRI) as first-line treatment for metastatic colorectal cancer: the Gruppo Oncologico Nord Ovest. J Clin Oncol. 2007;25:1670-6.

25. Souglakos J, Androulakis N, Syrigos K, et al. FOLFOXIRI (folinic acid, 5-fluorouracil, oxaliplatin and irinotecan) vs. FOLFIRI (folinic acid, 5-fluo-rouracil and irinotecan) as first-line treatment in metastatic colorectal cancer (MCC): a multicenter randomized phase III trial from the Hellenic Oncology Research Group (HORG). Br J Cancer. 2006;94:798-805.

26. Masi G, Loupakis F, Salvatore L, et al. Bevacizumab with FOLFOXIRI (irinotecan, oxaliplatin, fluorouracil and folinate) as first-line treatment for metastatic colorectal cancer: a phase 2 trial. Lancet Oncol. 2010;11:845-52.

27. Gunturu KS, Yao X, Cong X, Thumar JR, Hochster HS, Stein SM, Lacy J. FOLFIRINOX for locally advanced and metastatic pancreatic cancer: single institution retrospective review of efficacy and toxicity. Med Oncol. 2013;30:361.

28. Okusaka T, Ikeda M, Fukutomi A, et al. Phase II study of FOLFIRINOX for chemotherapy-naïve Japanese patients with metastatic pancreatic cancer. Cancer Sci. 2014;105:1321-6.

29. Li M, Wang Z, Guo J, et al. Clinical significance of UGT1A1 gene polymorphisms on irinotecan-based regimens as the treatment in metastatic colorectal cancer. Onco Targets Ther. 2014;7:1653-61.

30. Ramirez K, Koch M, Edenfield W. Irinotecan-induced dysarthria: a case report and review of the literature. J Oncol Pharm Pract. 2017. https://doi. org/10.1177/1078155216634181. 